\title{
The Thermocapillary Migrations of Two Bubbles in Microgravity Environment
}

\author{
Ren Sun ${ }^{1}$ and Wen-Rui Hu \\ National Microgravity Laboratory/CAS, Institute of Mechanics, Chinese Academy of Sciences, Beijing 100080, China
}

Received February 25, 2002; accepted July 16, 2002

\begin{abstract}
The thermocapillary motion of two bubbles along their line of centers in a uniform temperature gradient is investigated theoretically. The bubbles are moving in the direction of the temperature gradient. And the interaction between the leading bubble and the trailing one becomes significant as the separation distance between them is decreased greatly so that the bubble interaction is considered in this case. The appropriate equations of momentum and energy are solved using the method of reflections. In order to proceed analytically, sets of transformations between two coordinates are obtained. By using these transformations and the reflection process, accurate migration velocities of these two bubbles in the microgravity environment are derived for the limit of small Marangoni and Reynolds numbers. These results are employed to describe the thermocapillary motion of two bubbles and to estimate the effects of bubble size and the thermal gradient on the interaction between two bubbles. All of our results for the migration of the two bubbles demonstrate that the approach of the second bubble to the first one intensifies the mutual interaction between these two bubbles and yields some interesting thermocapillary motions. (c) 2002 Elsevier Science (USA)

Key Words: thermocapillary motion; two bubbles; migration velocity; thermal gradient; the reflection method.
\end{abstract}

\section{INTRODUCTION}

It has long been known that when a bubble is present in a continuous host fluid and subjected to a temperature gradient, it will move toward the hot portion of the host fluid in the microgravity environment. This is attributed to the temperatureinduced variation of the surface tension on the bubble interface. The consequence of the variation of surface tension due to a thermal gradient results in a tangential stress which, by viscous traction, causes the motion of the neighboring fluid. As a result, the bubble is itself propelled to the hot side of the host fluid. The investigation of the migration of bubbles in fluids is very important for basic research as well as for materials science and chemical engineering. Especially, the thermocapillary phenomena has received much attention with the development of the orbiting spacecraft and increasing prospects of experimenting and manufacturing in reduced-gravity environments.

${ }^{1}$ To whom correspondence should be addressed.E-mail: drrsun@yahoo.com.
The thermocapillary migration was first investigated by Young, Goldstein, and Block (1), who provided a theoretical description for the migration velocity of an isolated bubble in a bulk fluid with an imposed thermal gradient. This can be shown to hold when Reynolds and Marangoni numbers are small. They also observed the movement of a bubble in the liquid. The theory of Young, Goldstein, and Block was extended by Subramanian $(2,3)$ for the case with a convective heat transport in a microgravity environment. Reviews of the literature can be found in Wozniak et al. (4) and Subramanian (5).

In practical applications, collections of bubbles are usually encountered, and the interaction between them will be more important. In a previous article, Meyyappan et al. (6) theoretically investigated an axisymmetric thermocapillary migration of two bubbles in the microgravity environment moving along their line of centers in the quasi-static state, and the bispherical coordinate system is used. They found that the smaller one of the two bubbles always runs more rapidly than that in the isolated case, while the larger bubble moves slightly slower than that in the isolated case. As a corollary, the velocities of two bubbles with an identical diameter are the same and equal to the value in the case of an isolated bubble. This result was obtained numerically by Meyyappan et al., and the analytical one was showed later by Feuillebois (7). Meyyappan and Subramanian (8) analyzed the thermocapillary migration of two bubbles in arbitrary orientation with respect to an applied temperature gradient by using a far-field approximation, while Anderson (9) solved the problem of two arbitrarily oriented droplets by using the reflection method (10). Acrivos et al. (11) also predicted the thermocapillary migration velocities of a number of identical bubbles, and the results agree with the ones shown in Ref. 9. Wang et al. (12) used the method of twin multipole expansions to study the thermocapillary migration of two bubbles oriented arbitrarily with respect to the applied thermal gradient and obtain the solution in the form of infinite series with undetermined coefficients. They then truncated the series and obtained the coefficients to the desired accuracy.

The objective of this paper is to make a further investigation by another approach on the interaction between two bubbles and predict their thermocapillary motions. To make the analysis tractable, we also restrict our attention only to the case of small Reynolds and Marangoni numbers without considering 
the convective terms in the momentum equations and the energy equation. The process of a bubble pursuing another bubble is an unsteady one, but in many practical applications, such as the glass processing, the Reynolds number based on the bubble radius and the typical velocity, $u_{0}=T_{\mathrm{g}}\left|\gamma^{\prime}\right| R / \mu$, is usually low. Here $R$ is the radius of a bubble, $T_{\mathrm{g}}$ the thermal gradient imposed in the fluid, $\mu$ the dynamic viscosity of the continuous phase, and $\gamma^{\prime}$ refers to the rate of change of interfacial tension with temperature. In the scaled momentum equation $\operatorname{Re}[\partial \mathbf{u} / \partial t+\mathbf{u} \cdot \nabla \mathbf{u}]=-\nabla p+\nabla^{2} \mathbf{u}$, as indicated by Meyyappan et al. (6), if the Reynolds number is low and the variation of the velocity with time due to the bubble migration is not large, the Stokes equation will give a better approximation of the thermocapillary motion of two bubbles pursuing one by another.

An analytical method is used in the present paper by establishing two coordinate systems fixed respectively at the centers of the two bubbles. By using the method of reflections, sets of transformations are adopted to obtain singularity combinations satisfying the impenetrable and the adiabatic conditions on the bubble surfaces. In light of these combinations, explicit thermocapillary migration velocities of the two bubbles satisfying the balance of tangential stress on the bubble surfaces are constructed from the expression for a single bubble. These expressions are iterative ones in form, and furthermore, they are just power series of given bubble radii and the separation distance. Therefore, this is the numerical advantage of the approach.

The governing equations and boundary conditions are presented in section 2 . In section 3 , we are concerned with the transformations of some harmonics. This is followed by analysis of axisymmetric thermocapillary migration of the two bubbles, including the details that are necessary to complete this portion of the analysis. An explicit recurrence formula to calculate the migration velocities will be developed. In section 4 , the prediction of thermocapillary motions of the two bubbles is given in light of the computational results, and several examples are discussed. Finally, conclusions are presented in section 5.

\section{GOVERNING EQUATIONS AND BOUNDARY CONDITIONS}

The axisymmetric thermocapillary motions of two spherical bubbles of radii $R_{1}$ and $R_{2}$, respectively, are considered in an unbounded host fluid in the microgravity environment. These two bubbles move along their line of centers in the positive $z$ direction, as shown in Fig. 1. In the continuous phase, a temperature field, which has a uniform thermal gradient $T_{\mathrm{g}}$ parallel to the $z$-axis far away from the pair of bubbles, is imposed. A leading bubble with center $o_{1}$ located at $z_{1}$ moves with a migration velocity $u_{1}$, while a trailing bubble with center $o_{2}$ situated at $z_{2}$ moves with velocity $u_{2}$. The separation distance between the bubble centers $o_{1} o_{2}$ is denoted by $s$.

For a two-bubble system, two coordinates are introduced for convenience, one is a Cartesian coordinate $(x, y, z)$ fixed at the center of bubble 1 , the other $(X, Y, Z)$ at the center of bubble 2 , as shown in Fig. 2. The translational transformation between

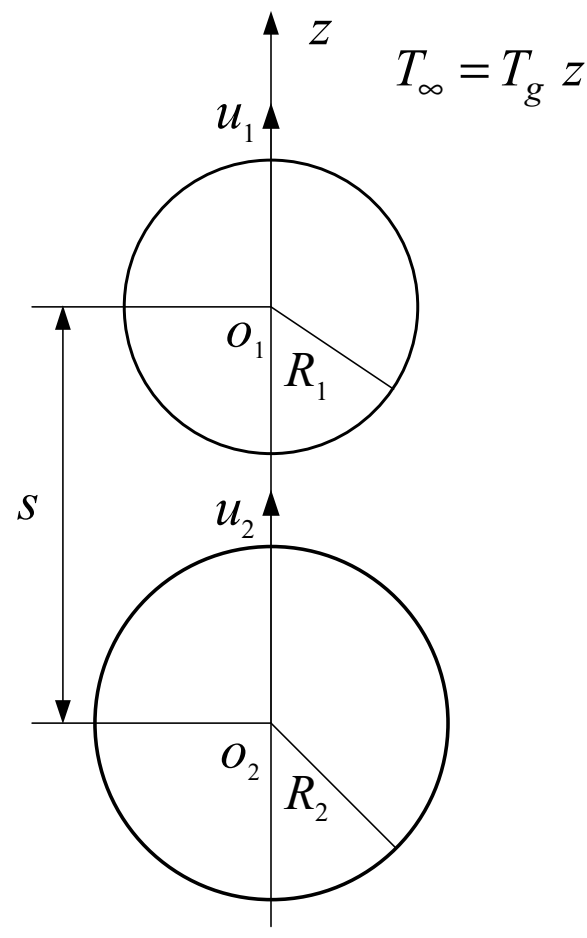

FIG. 1. Schematic of the leading and trailing bubbles.

$(x, y, z)$ and $(X, Y, Z)$ is

$$
\left\{\begin{array}{l}
x=X \\
y=Y \\
z=Z-s .
\end{array}\right.
$$

These two Cartesian coordinate systems can be turned into

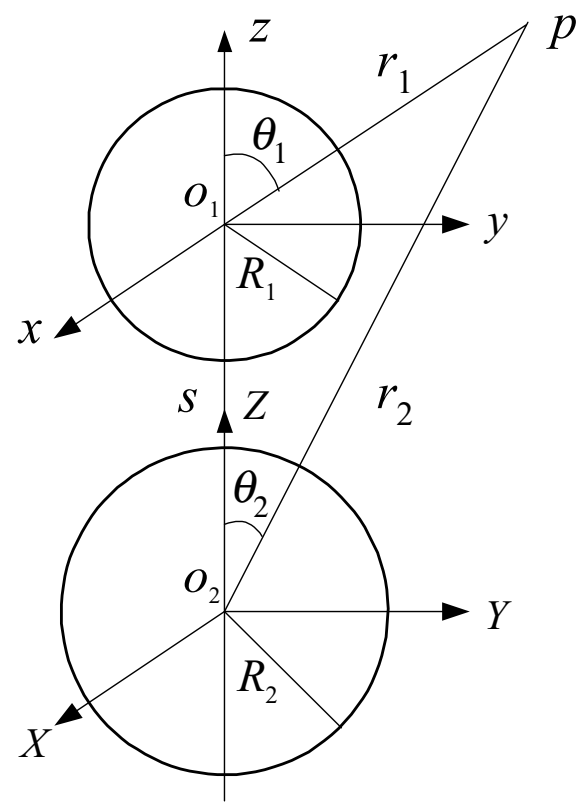

FIG. 2. Sketch of the two bubbles and the corresponding coordinate systems. 
spherical ones by

$$
\left\{\begin{array} { l } 
{ x = r _ { 1 } \operatorname { s i n } \theta _ { 1 } \operatorname { c o s } \varphi } \\
{ y = r _ { 1 } \operatorname { s i n } \theta _ { 1 } \operatorname { s i n } \varphi } \\
{ z = r _ { 1 } \operatorname { c o s } \theta _ { 1 } }
\end{array} \text { and } \quad \left\{\begin{array}{l}
X=r_{2} \sin \theta_{2} \cos \varphi \\
Y=r_{2} \sin \theta_{2} \sin \varphi \\
Z=r_{2} \cos \theta_{2}
\end{array}\right.\right.
$$

Assuming that gravitational effects are ignored, the deformation of the bubbles is negligible, and thus the normal stress balance on the bubble surfaces is ignored. In addition, the density, viscosity, and thermal conductivity of the gas phase are negligibly small in comparison with those in the host fluid, and all other physical properties are assumed independent of temperature except for the surface tension, $\gamma$, which varies linearly with temperature $\left(\gamma=\gamma_{0}-\gamma^{\prime} T\right)$. The assumption on the very low thermal conductivity of the gas phase means the normal flux of energy on each bubble surface is zero, and the temperature distribution has a jump across the surface of these bubbles. As mentioned earlier, for highly viscous glass melts, our attention in this analysis is focused only on the case in which the Reynolds and Marangoni numbers are set equal to zero (Meyyappan et al. (6)). Hence the governing equations in the microgravity environment can be written as

$$
\begin{aligned}
& \nabla \cdot \mathbf{u}=0, \\
& \nabla p-\mu \nabla^{2} \mathbf{u}=0, \\
& \nabla^{2} T=0 .
\end{aligned}
$$

Here $\mathbf{u}$ denotes the velocity vector of the host fluid and $T$ is the temperature field. Here $T=T_{\mathrm{g}} z+T^{\prime}$ is the summation of a linear temperature field far away from the bubbles and a disturbance field.

The boundary conditions to be satisfied on the surface of the bubble $i$ are

$$
\begin{aligned}
& \mathbf{u} \cdot \mathbf{n}_{i}=u_{i} \mathbf{e}_{3} \cdot \mathbf{n}_{i}, \\
& \boldsymbol{\tau}_{i}-\left.\nabla \gamma\right|_{\Sigma_{i}}=0, \\
& \mathbf{n}_{i} \cdot \nabla T=0,
\end{aligned}
$$

and

$$
\mathbf{u} \rightarrow 0, \quad T \rightarrow T_{\mathrm{g}} z, \quad \text { at infinity }
$$

where $\mathbf{n}_{i}$ is the unit vector normal to the surface of bubble $i, \boldsymbol{\tau}_{i}$ its shear stress on the same surface $\Sigma_{i}$, and $\mathbf{e}_{3}$ denotes the unit vector in the positive $z$-direction. Boundary condition [4a] denotes impenetrability on the surfaces of the bubbles; [4b] represents the balance of tangential stress on the bubble interfaces, and [4c] is the condition of a zero heat flux on each bubble surface.

\section{ANALYTICAL SOLUTION}

Based on the general solution given by Lamb (13), the velocity field for a single bubble of radius $R$ in a thermocapillary migration with velocity $u_{0}$ along the $z$-axis is written in the form of

$$
\mathbf{u}=-\frac{u_{0} R^{3}}{2} \nabla\left(\frac{z}{r^{3}}\right)+\frac{a}{2 \mu}\left(R^{2}-\frac{r^{2}}{3}\right) \nabla\left(\frac{z}{r^{3}}\right)+\frac{2 a \nabla z}{3 \mu r}
$$

where $\mu$ denotes the dynamic viscosity of the fluid, $a$ is a constant to be determined by the boundary condition. In the gravitational field, $a=g R^{3} \rho / 3$, where $g$ is the gravitational acceleration and $\rho$ the density of the host fluid, as shown in Ref. 1. In the microgravity environment, $g \rightarrow 0$ leads to $a \rightarrow 0$.

Similarly, the temperature field for the same bubble neglecting the normal flux of energy is

$$
T=T_{\mathrm{g}}\left(r+\frac{R^{3}}{2 r^{2}}\right) \cos \theta .
$$

Owing to the axisymmetric nature of the system, there is no dependence of the field variables on the azimuthal coordinate $\varphi$.

\section{Transformations between Harmonics}

Because it is impossible to find the solution for the problem with a triply connected region by using a limited number of simple functions, the method of reflections has to be adopted. For this reason, we look for transformations between two coordinate systems. A pair of transformations of associated Legendre functions between $\left(r_{1}, \theta_{1}, \varphi\right)$ and $\left(r_{2}, \theta_{2}, \varphi\right)$ was given by Hobson (14) as

$$
\frac{P_{n}^{m}}{r_{1}^{n+1}}=(-1)^{n-m} \sum_{k=m}^{\infty} C_{m n k} r_{2}^{k} \mathrm{P}_{k}^{m}
$$

and

$$
\frac{\mathrm{P}_{n}^{m}}{r_{2}^{n+1}}=\sum_{k=m}^{\infty}(-1)^{k-m} C_{m n k} r_{1}^{k} P_{k}^{m}
$$

where $C_{m n k}=\left(\begin{array}{c}n+k \\ n-m\end{array}\right) / s^{n+k+1}(n \geq m)$. Here $P_{n}^{m}$ and $\mathrm{P}_{n}^{m}$ denote $P_{n}^{m}\left(\cos \theta_{1}\right)$ and $P_{n}^{m}\left(\cos \theta_{2}\right)$, respectively.

\section{Derivation of Bubble Velocities}

In this subsection, we solve the problem of two interacting bubbles using a reflection procedure corresponding to that presented by Sun and Chwang $(15,16)$. Suppose that the initial disturbance velocity generated by bubble 1 is given by $\mathbf{u}_{0}^{\prime}$ and in the $\left(r_{1}, \theta_{1}, \varphi\right)$ spherical coordinates takes the form

$$
\mathbf{u}_{0}^{\prime(1)}=\nabla\left(\frac{P_{1}}{r_{1}^{2}}\right) \text {. }
$$

Here superscripts (1) and (2) mean that physical variables are expressed in the $\left(r_{1}, \theta_{1}, \varphi\right)$ and $\left(r_{2}, \theta_{2}, \varphi\right)$ coordinate system, respectively. 
When a second bubble is released at a standstill into the flow field at $o_{2}$, around bubble 2, $\mathbf{u}_{0}^{\prime}$ can be expressed by using the transformation in [7a] as

$$
\mathbf{u}_{0}^{\prime(2)}=-\sum_{k=0}^{\infty} C_{01 k} \nabla\left(r_{2}^{k} \mathrm{P}_{k}\right)=\sum_{k=0}^{\infty} \lambda_{1 k}^{(1)} \nabla\left(r_{2}^{k} \mathrm{P}_{k}\right),
$$

where $\lambda_{1 k}^{(1)}=-C_{01 k}$. In order to satisfy the impenetrable boundary condition on the surface of bubble 2 , we should add an extra velocity $\mathbf{u}_{1}^{\prime}$

$$
\mathbf{u}_{1}^{\prime(2)}=\sum_{k=0}^{\infty} \frac{k}{(k+1)} \lambda_{1 k}^{(1)} R_{2}^{2 k+1} \nabla\left(\frac{\mathrm{P}_{k}}{r_{2}^{k+1}}\right)
$$

into the flow field. In light of transformation [7b], $\mathbf{u}_{1}^{\prime}$ may be expressed in the $\left(r_{1}, \theta_{1}, \varphi\right)$ coordinates as

$$
\begin{aligned}
\mathbf{u}_{1}^{\prime(1)} & =\sum_{k=0}^{\infty} \sum_{l=0}^{\infty} \frac{(-1)^{k} l R_{2}^{2 l+1}}{(l+1)} \lambda_{1 l}^{(1)} C_{0 l k} \nabla\left(r_{1}^{k} P_{k}\right) \\
& =\sum_{k=0}^{\infty} \lambda_{1 k}^{(2)} \nabla\left(r_{1}^{k} P_{k}\right),
\end{aligned}
$$

where $\lambda_{1 k}^{(2)}=(-1)^{k} \sum_{l=0}^{\infty} l R_{2}^{2 l+1} \lambda_{1 l}^{(1)} C_{0 l k} /(l+1)$, and the added disturbance makes an extra contribution to the velocity field and violates the impenetrable condition on bubble 1 . This disturbance should be counteracted by introducing another correcting velocity. Repeating the same procedure in succession leads to an infinite sequence of reflection velocities. And thus the velocity field for the two-bubble system in relation to $\mathbf{u}_{0}^{\prime}$ is

$$
\begin{aligned}
\mathbf{u}^{\prime}= & \nabla\left(\frac{P_{1}}{r_{1}^{2}}\right)+\sum_{k=0}^{\infty} \frac{k R_{2}^{2 k+1}}{k+1} \nabla\left(\frac{\mathrm{P}_{k}}{r_{2}^{k+1}}\right) \sum_{i=0}^{\infty} \lambda_{1 k}^{(2 i+1)} \\
& +\sum_{k=0}^{\infty} \frac{k R_{1}^{2 k+1}}{k+1} \nabla\left(\frac{P_{k}}{r_{1}^{k+1}}\right) \sum_{i=0}^{\infty} \lambda_{1 k}^{(2 i)}
\end{aligned}
$$

where $\lambda_{1 k}^{(i)}$ is in the form of recurrence formulae

$$
\begin{aligned}
\lambda_{1 k}^{(0)} & =0, \quad \lambda_{1 k}^{(1)}=-C_{01 k}, \\
\lambda_{1 k}^{(2 i)} & =(-1)^{k} \sum_{l=0}^{\infty} \frac{l R_{2}^{2 l+1} C_{0 l k} \lambda_{1 l}^{(2 i-1)}}{l+1}, \\
\lambda_{1 k}^{(2 i+1)} & =\sum_{l=0}^{\infty} \frac{(-1)^{l} l R_{1}^{2 l+1} C_{0 l k} \lambda_{1 l}^{(2 i)}}{l+1} .
\end{aligned}
$$

The expressions above hold for all positive integers $k$ and $i$.

Next, let us consider the contribution to the velocity field just from an initial disturbance velocity caused by bubble 2 when bubble 1 is inserted motionless nearby. Similarly, the velocity field for the system in relation to the disturbance velocity $\mathbf{u}_{0}^{\prime \prime}$, which in the $\left(r_{2}, \theta_{2}, \varphi\right)$ coordinates is written as

$$
\mathbf{u}_{0}^{\prime \prime(2)}=\nabla\left(\frac{\mathrm{P}_{1}}{r_{2}^{2}}\right),
$$

is expressed as

$$
\begin{aligned}
\mathbf{u}^{\prime \prime}= & \nabla\left(\frac{\mathrm{P}_{1}}{r_{2}^{2}}\right)+\sum_{k=0}^{\infty} \frac{k R_{1}^{2 k+1}}{k+1} \nabla\left(\frac{P_{k}}{r_{1}^{k+1}}\right) \sum_{i=0}^{\infty} \Lambda_{1 k}^{(2 i+1)} \\
& +\sum_{k=0}^{\infty} \frac{k R_{2}^{2 k+1}}{k+1} \nabla\left(\frac{\mathrm{P}_{k}}{r_{2}^{k+1}}\right) \sum_{i=0}^{\infty} \Lambda_{1 k}^{(2 i)},
\end{aligned}
$$

where

$$
\begin{aligned}
\Lambda_{1 k}^{(0)} & =0, \quad \Lambda_{1 k}^{(1)}=(-1)^{k} C_{01 k}, \\
\Lambda_{1 k}^{(2 i)} & =\sum_{l=0}^{\infty} \frac{(-1)^{l} l R_{1}^{2 l+1} C_{0 l k} \Lambda_{1 l}^{(2 i-1)}}{l+1}, \\
\Lambda_{1 k}^{(2 i+1)} & =(-1)^{k} \sum_{l=0}^{\infty} \frac{l R_{2}^{2 l+1} C_{0 l k} \Lambda_{1 l}^{(2 i)}}{l+1},
\end{aligned}
$$

holding for all positive integers $k$ and $i$.

For an initial disturbance temperature field created by bubble 1,

$$
T_{0}^{(1)}=\frac{P_{1}}{r_{1}^{2}},
$$

then the temperature distribution for the two-bubble system becomes

$$
\begin{aligned}
T^{\prime}= & \frac{P_{1}}{r_{1}^{2}}+\sum_{k=0}^{\infty} \frac{k R_{2}^{2 k+1}}{(k+1)} \frac{\mathrm{P}_{k}}{r_{2}^{k+1}} \sum_{i=0}^{\infty} \phi_{k}^{(2 i+1)} \\
& +\sum_{k=0}^{\infty} \frac{k R_{1}^{2 k+1}}{(k+1)} \frac{P_{k}}{r_{1}^{k+1}} \sum_{i=0}^{\infty} \phi_{k}^{(2 i)}
\end{aligned}
$$

where

$$
\begin{aligned}
\phi_{k}^{(0)} & =0, \quad \phi_{k}^{(1)}=-C_{01 k}, \\
\phi_{k}^{(2 i)} & =(-1)^{k} \sum_{l=0}^{\infty} \frac{l R_{2}^{2 l+1} C_{0 l k} \phi_{l}^{(2 i-1)}}{l+1}, \\
\phi_{k}^{(2 i+1)} & =\sum_{l=0}^{\infty} \frac{(-1)^{l} l R_{1}^{2 l+1} C_{0 l k} \phi_{l}^{(2 i)}}{l+1},
\end{aligned}
$$

whereas for the initial disturbance temperature field aroused by bubble 2 ,

$$
T_{0}^{\prime \prime(2)}=\frac{\mathrm{P}_{1}}{r_{2}^{2}}
$$


the temperature distribution for the whole system is

$$
\begin{aligned}
\mathrm{T}^{\prime \prime}= & \frac{\mathrm{P}_{1}}{r_{2}^{2}}+\sum_{k=0}^{\infty} \frac{k R_{1}^{2 k+1}}{(k+1)} \frac{P_{k}}{r_{1}^{k+1}} \sum_{i=0}^{\infty} \Phi_{k}^{(2 i+1)} \\
& +\sum_{k=0}^{\infty} \frac{k R_{2}^{2 k+1}}{(k+1)} \frac{\mathrm{P}_{k}}{r_{2}^{k+1}} \sum_{i=0}^{\infty} \Phi_{k}^{(2 i)}
\end{aligned}
$$

where

$$
\begin{gathered}
\Phi_{k}^{(0)}=0, \quad \Phi_{k}^{(1)}=(-1)^{k} C_{01 k}, \\
\Phi_{k}^{(2 i)}=\sum_{l=0}^{\infty} \frac{(-1)^{l} l R_{1}^{2 l+1} C_{0 l k} \Phi_{l}^{(2 i-1)}}{l+1} \\
\Phi_{k}^{(2 i+1)}=(-1)^{k} \sum_{l=0}^{\infty} \frac{l R_{2}^{2 l+1} C_{0 l k} \Phi_{l}^{(2 i)}}{l+1} .
\end{gathered}
$$

According to the superposition theorem for linear equations, the velocity field in surrounding fluid is $\mathbf{u}=-u_{1} R_{1}^{3} \mathbf{u}^{\prime} / 2-$ $u_{2} R_{2}^{3} \mathbf{u}^{\prime \prime} / 2$, and the temperature distribution takes the form $T=T_{\mathrm{g}}\left(z+R_{1}^{3} T^{\prime} / 2+R_{2}^{3} T^{\prime \prime} / 2\right)$. Substituting $\mathbf{u}$ and $T$ above into [4b] leads, with manipulation, to two explicit expressions of the bubble migration velocity in the microgravity environment,

$$
\begin{aligned}
& \frac{u_{1}}{R_{1}}\left\{1+\frac{R_{1}^{3}}{2} \sum_{i=0}^{\infty} \lambda_{11}^{(2 i)}\right\}+\frac{R_{2}^{3} u_{2}}{2 R_{1}} \sum_{i=0}^{\infty} \Lambda_{11}^{(2 i+1)} \\
& -\frac{\gamma^{\prime} T_{\mathrm{g}}}{2 \mu}\left\{1+\frac{R_{1}^{3}}{2} \sum_{i=0}^{\infty} \phi_{1}^{(2 i)}+\frac{R_{2}^{3}}{2} \sum_{i=0}^{\infty} \Phi_{1}^{(2 i+1)}\right\}=0
\end{aligned}
$$

and

$$
\begin{aligned}
& \frac{u_{2}}{R_{2}}\left\{1+\frac{R_{2}^{3}}{2} \sum_{i=0}^{\infty} \Lambda_{11}^{(2 i)}\right\}+\frac{R_{1}^{3} u_{1}}{2 R_{2}} \sum_{i=0}^{\infty} \lambda_{11}^{(2 i+1)} \\
& -\frac{\gamma^{\prime} T_{\mathrm{g}}}{2 \mu}\left\{1+\frac{R_{2}^{3}}{2} \sum_{i=0}^{\infty} \Phi_{1}^{(2 i)}+\frac{R_{1}^{3}}{2} \sum_{i=0}^{\infty} \phi_{1}^{(2 i+1)}\right\}=0 .
\end{aligned}
$$

Readers can obtain well-known results, $u_{i}=R_{i} \gamma^{\prime} T_{\mathrm{g}} /(2 \mu)$, by letting $s \rightarrow \infty$ in Eqs. [19a] and [19b].

\section{RESULTS AND DISCUSSION}

The migration velocity expressions coupled with temperature field, Eqs. [19a] and [19b], are applied to predict the thermocapillary migration of the two axisymmetric moving bubbles in the quasi-static process. To do this, we have to resort to the numerical computation of migration velocity expressions since iterative formulae in Eqs. [19a] and [19b] are not directly available in general. As each of the $m$ th term in recurrence formulae [12], [14], [16], and [18] behaves like $1 / s^{3 m}$, the truncated series in the above formulae at $m=50$ would have errors smaller than the error tolerance of five significant figures even for a near contact. The series in [19a] and [19b] truncated at $i=50$ make our numerical results accurate up to the 300th inverse power of $s$ in the present paper. To assure convergence, our calculations are carried out with automatic increase in $i$ until the results remain unchanged to the desired level of accuracy. In addition, to obtain trajectories of the bubbles, at first the trailing bubble is released at the origin, i.e., $z_{20}=0$, and the leading bubble is put at $z_{10}$ simultaneously. At the beginning of the $j$ th time interval $\delta t_{j}(j=1,2 \ldots)$, the migration velocities of the given bubbles, $u_{1 j}$ and $u_{2 j}$, are obtained by solving algebraic Eqs. [19a] and $[19 \mathrm{~b}]$. At the end of the $j$ th time interval, the new position of the center of bubble $i$ is simply calculated by $z_{i j}=z_{i j-1}+$ $u_{i j} \delta t_{j}$.

To better describe migration velocities of the two bubbles, we define the interaction parameter as in Ref. (6) with a little correction:

$$
\begin{aligned}
\Omega_{i}\left(S, \lambda_{i}\right) & =\frac{u_{i}}{\max \left(u_{1 \mathrm{YGB}}, u_{2 \mathrm{YGB}}\right)}, \\
S & =\frac{s}{\max \left(R_{1}, R_{2}\right)}, \\
\lambda_{i} & =\frac{R_{i}}{\max \left(R_{1}, R_{2}\right)},
\end{aligned}
$$

where $u_{i Y G B}=R_{i} \gamma^{\prime} T_{\mathrm{g}} /(2 \mu)$.

Let us first consider the axisymmetric thermocapillary migration of two identical bubbles along their line of centers. Based on Eqs. [19a] and [19b], interaction parameters $\Omega_{1}$ and $\Omega_{2}$ against the scaled separation distance $S$ are plotted in Fig. 3 for equalsized bubbles. Note from the figure that the two equal-sized bubbles move at the velocity that they would have if they were in isolation, independent of the separation distance, as indicated by Meyyappan et al. This result may be explained quantitatively as follows: as $R_{1}=R_{2}=R$, Eqs. [19a] and [19b] can be

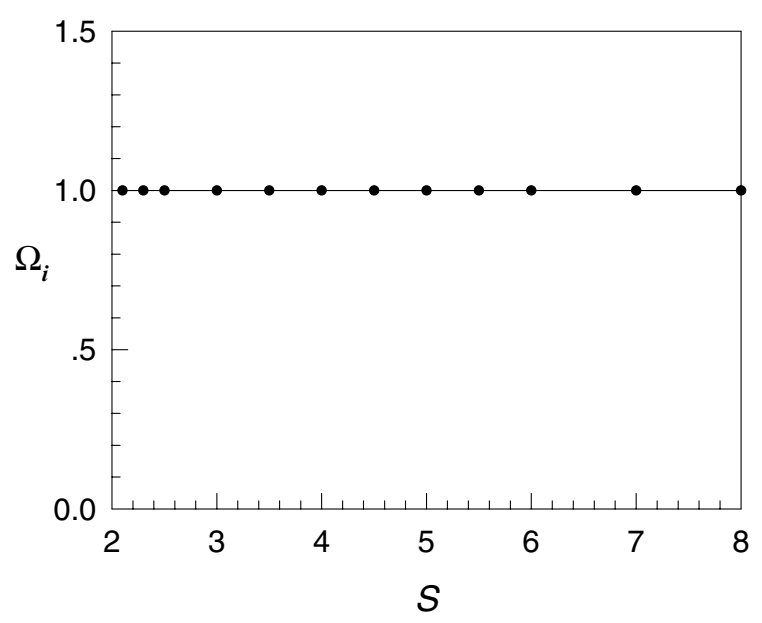

FIG. 3. Interaction parameters, $\Omega_{1}$ and $\Omega_{2}$, for two identical bubbles versus the dimensionless separation distance $S$. 
rewritten as

$$
\begin{aligned}
& u_{1}\left\{1+\frac{R^{3}}{2} \sum_{i=0}^{\infty} \lambda_{11}^{(2 i)}\right\}+\frac{R^{3} u_{2}}{2} \sum_{i=0}^{\infty} \Lambda_{11}^{(2 i+1)} \\
& -\frac{R \gamma^{\prime} T_{\mathrm{g}}}{2 \mu}\left\{1+\frac{R^{3}}{2} \sum_{i=0}^{\infty} \phi_{1}^{(2 i)}+\frac{R^{3}}{2} \sum_{i=0}^{\infty} \Phi_{1}^{(2 i+1)}\right\}=0
\end{aligned}
$$

and

$$
\begin{aligned}
& u_{2}\left\{1+\frac{R^{3}}{2} \sum_{i=0}^{\infty} \Lambda_{11}^{(2 i)}\right\}+\frac{R^{3} u_{1}}{2} \sum_{i=0}^{\infty} \lambda_{11}^{(2 i+1)}-\frac{R \gamma^{\prime} T_{\mathrm{g}}}{2 \mu} \\
& \quad \times\left\{1+\frac{R^{3}}{2} \sum_{i=0}^{\infty} \Phi_{1}^{(2 i)}+\frac{R^{3}}{2} \sum_{i=0}^{\infty} \phi_{1}^{(2 i+1)}\right\}=0 .
\end{aligned}
$$

From iterative formulae [12], [14], [16], and [18], it is easy to find that

$$
\begin{array}{ll}
\sum_{i=0}^{\infty} \lambda_{11}^{(2 i)}=\sum_{i=0}^{\infty} \phi_{1}^{(2 i)}, & \sum_{i=0}^{\infty} \Lambda_{11}^{(2 i)}=\sum_{i=0}^{\infty} \Phi_{1}^{(2 i)} \\
\sum_{i=0}^{\infty} \lambda_{11}^{(2 i+1)}=\sum_{i=0}^{\infty} \phi_{1}^{(2 i+1)}, & \sum_{i=0}^{\infty} \Lambda_{11}^{(2 i+1)}=\sum_{i=0}^{\infty} \Phi_{1}^{(2 i+1)} .
\end{array}
$$

Therefore, the conclusion is $u_{1}=u_{2}=R \gamma^{\prime} T_{\mathrm{g}} /(2 \mu)$ by solving [23a] and [23b]. This means that the thermal and fluid mechanical interaction effects are counterbalanced thoroughly.

Using the expressions given in Eqs. [19a] and [19b], we can determine the influence of the interaction on the migration of the two bubbles in the course of a large trailing bubble pursuing a small leading one. Figures 4 and 5 show the interaction parameters $\Omega_{1}$ and $\Omega_{2}$ vary with the dimensionless separation distance $S$ for four different size ratios $R_{1} / R_{2}=0.5,0.2,0.1$, and 0.01 . It is noted from Fig. 4 that the interaction between two bubbles

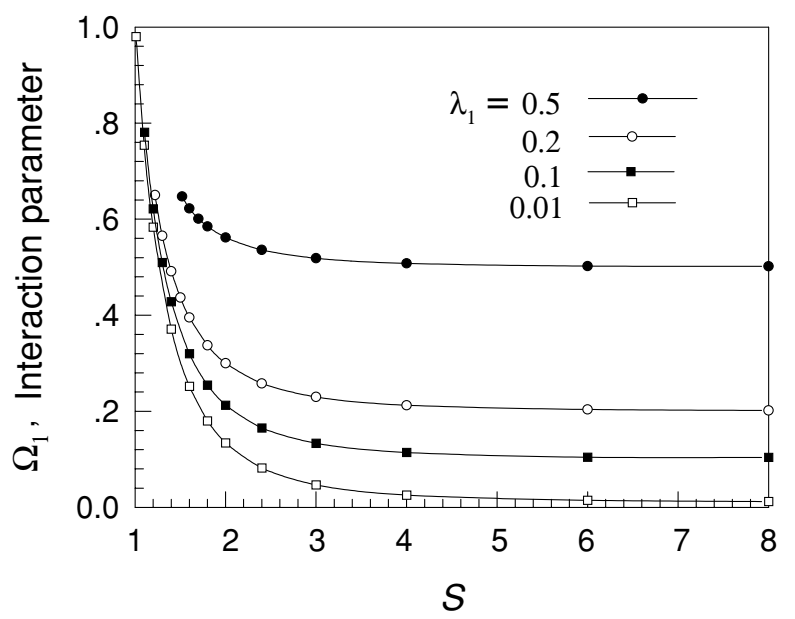

FIG. 4. Interaction parameter, $\Omega_{1}$, for the smaller bubble versus the dimensionless separation distance $S$.

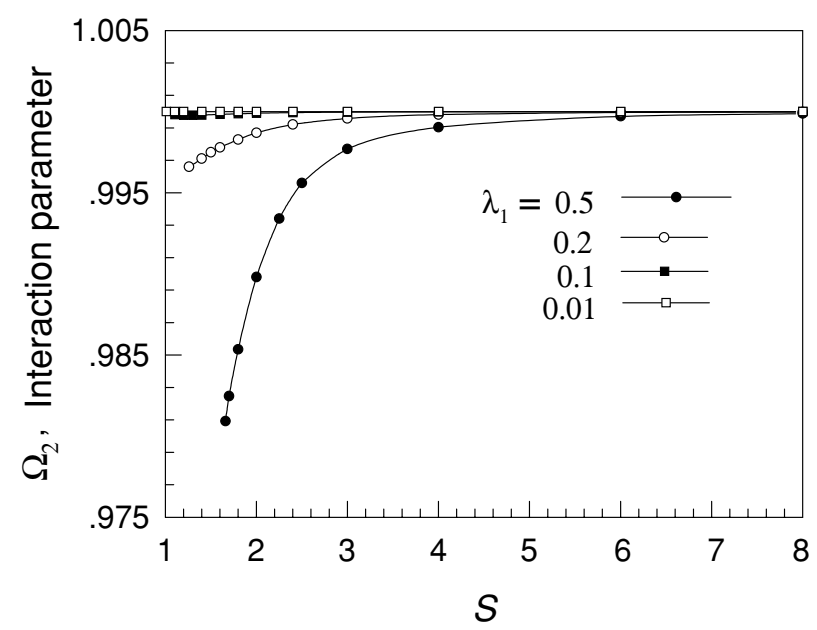

FIG. 5. Interaction parameter, $\Omega_{2}$, for the larger bubble versus the dimensionless separation distance $S$.

has significant influence on the migration of the smaller bubble. The greater the difference in size between the two bubbles, the greater the increase in the velocity of the smaller one. In the case of $R_{1} / R_{2}=0.01$, the velocity of the small leading bubble would reach a maximum that is 97 times more than that in the isolated case and that nearly equals that of the large trailing one. As a corollary, for a very small leading bubble, if there is no bubble break, the two bubbles would ultimately move forward at the same velocity. One can see a qualitative explanation below: as $R_{1} \rightarrow 0$ and $s \rightarrow R_{1}+R_{2} \rightarrow R_{2}$, Eqs. [19a] and [19b] can be simplified as

$$
u_{1}-u_{2}+O\left(R_{1}\right)=0
$$

and

$$
u_{2}-\frac{R_{2} \gamma^{\prime} T_{\mathrm{g}}}{2 \mu}+o\left(R_{1}\right)=0
$$

that is, $u_{1} \approx u_{2} \approx R_{2} \gamma^{\prime} T_{\mathrm{g}} /(2 \mu)$. On the other hand, as $R_{1} \rightarrow 0$, one can derive again the Meyyappan heuristic approximation, $u_{1} / u_{1 \mathrm{YGB}}=1+\left(R_{2} / s\right)^{3}\left(R_{2} / R_{1}-1\right)$, directly from [19a] and [19b]. And even more important, it is found from the figure that as the dimensionless separation distance $S$ is less than 3.0, the migration velocity of the smaller bubble changes greatly due to interaction. This implies that there is an "interaction interval" around a bubble. The interaction interval is defined as one diameter apart from the bubble. If a second bubble intrudes into the interaction interval of the first one, its migration would be greatly affected by the first bubble. This phenomenon is consistent with the physical intuition.

Figure 5 shows the influence of the interaction on the migration of the larger bubble. It is seen from the figure that the effect of the interaction on the large bubble is not significant. The difference in velocity from a case of two touching bubbles to the separated one is less than $2 \%$. Therefore, one can think 


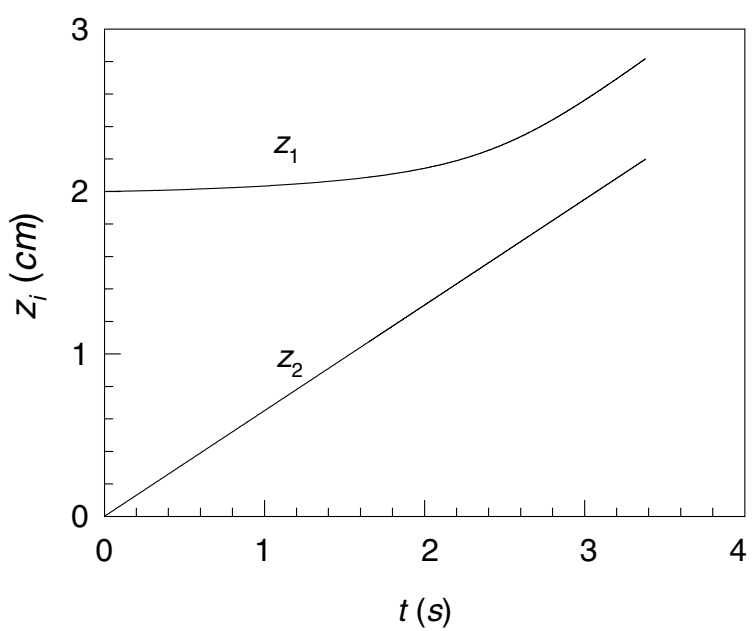

FIG. 6. Motions of two bubbles with $R_{1}=0.005 \mathrm{~cm}, R_{2}=0.5 \mathrm{~cm}$, $\gamma^{\prime}=0.034 \mathrm{dyn} / \mathrm{cm} \cdot \mathrm{K}, T_{\mathrm{g}}=30 \mathrm{~K} / \mathrm{cm}$, and $\mu=0.397 \mathrm{dyn} \cdot \mathrm{s} / \mathrm{cm}^{2}$.

that there is a negligible influence on the migration of the large bubble.

By comparing the results in Figs. 4 and 5 with ones given by Meyyappan et al., it is observed that for the corresponding size ratios, $R_{1} / R_{2}=0.5,0.2$, and 0.1 , the results agree well with each other except for rather small separation distances with a largest difference less than $0.3 \%$ over all the size ratios. The reason may be that these two solutions are expressed in terms of two kinds of functions respectively, hyperbolic functions and power ones, and thus there is a different accumulative error in each individual calculation. Power series of given bubble radii and the separation distance have a smaller accumulative error than those of hyperbolic functions, especially for touching bubble cases. But this slight difference is unrelated to the description of the physical process.

Figure 6 gives an example to illustrate the process of the large trailing bubble pursuing the small leading one. In this case, $R_{1}=0.005 \mathrm{~cm}, R_{2}=0.5 \mathrm{~cm}, \gamma^{\prime}=0.034 \mathrm{dyn} / \mathrm{cm} \cdot \mathrm{K}$, $T_{\mathrm{g}}=30 \mathrm{~K} / \mathrm{cm}$, and $\mu=0.397 \mathrm{dyn} \cdot \mathrm{s} / \mathrm{cm}^{2}$, corresponding to a case of two bubbles in vegetable oil. The two bubbles are first released at $z_{10}=1.5 \mathrm{~cm}$ and $z_{20}=0 \mathrm{~cm}$, respectively. Note that the small leading bubble gradually increases its velocity in the initial phase of migration and speeds up as $t=2.0 \mathrm{~s}$ when the separation distance is about $0.85 \mathrm{~cm}$. At last the two bubbles move forward at almost the same velocity while touching each other.

Based on Eqs. [19a] and [19b] and recurrence formulae [12], [14], [16], and [18], it is not difficult to find that if we exchange the values of $R_{1}$ and $R_{2}, u_{1}$ and $u_{2}$ have the same change, too. This demonstrates that all results are unchanged in spite of the order of alignment of the two bubbles.

\section{CONCLUSIONS}

This paper presents an analytical method of investigating an axisymmetric thermocapillary migration of two bubbles in the quasi-static limit. By using reflections, the analytical solution is derived. This solution is employed to predict the motion of two pursuing bubbles in the microgravity environment. The results indicate that the interaction between two bubbles has significant influence on the migration of the smaller bubble. This effect mainly is focused in an "interaction interval," which is about one diameter apart from the larger bubble. If a small bubble intrudes into the zone, its velocity changes greatly due to the interaction. As a very small bubble is in this case close to another large one, they would ultimately move forward at the same velocity if there is no bubble break.

\section{ACKNOWLEDGMENT}

This work was supported in part by the National Natural Science Foundation under Grant 19789201.

\section{REFERENCES}

1. Young, N. O., Goldstein, J. S., and Block, M. J., J. Fluid Mech. 6, 350 (1959).

2. Subramanian, R. S., AIChE J. 27, 646 (1981).

3. Subramanian, R. S., “Advances in Space Research.” Pergamon, London, 1983.

4. Wozniak, G., Siekmann, J., and Srulijes, J., Z. Flugwiss. Weltraumforsch. 12, 137 (1988).

5. Subramanian, R. S., "Transport Processes in Bubbles, Drops and Particles." Hemisphere, New York, 1992.

6. Meyyappan, M., Wilcox, W. R., and Subramanian, R. S., J. Colloid Interface Sci. 94, 243 (1983).

7. Feuillebois, F., J. Colloid Interface Sci. 131, 267 (1989).

8. Meyyappan, M., and Subramanian, R. S., J. Colloid Interface Sci. 97, 219 (1984).

9. Anderson, J. L., Int. J. Multiphase Flow 11, 813 (1985).

10. Kim, S., and Karrila, S. J., "Microhydrodynamics: Principles and Selected Applications." Butterworth-Heinemann, New York, 1991.

11. Acrivos, A., Jeffrey, D. J., and Saville, D. A., J. Fluid Mech. 212, 95 (1990).

12. Wang, Y., Mauri, R., and Acrivos, A., J. Fluid Mech. 261, 47 (1994).

13. Lamb, H., "Hydrodynamics." Cambridge University Press, 1932.

14. Hobson, E. W., "The Theory of Spherical and Ellipsoidal Harmonics." Chelsea, New York, 1955.

15. Sun, R., and Chwang, A. T., J. Phys. Soc. Jpn. 70, 91 (2001).

16. Sun, R., and Chwang, A. T., Theor. Comp. Fluid Dyn. 15, 11 (2001). 